\title{
Création de
}

\section{la Revue Générale Nucléaire}

Notre initiative est-elle justifiée? Vient-elle au bon moment? Rencontrerat-elle un écho favorable? En publiant le premier numéro de la REVUE GENEERALE NUCLEAIRE, nous ne pouvons manquer d'agiter en nous-mêmes ces quelques interrogations. Sans attendre pour le moment de réponse... Créer une revue nouvelle, c'est toujours engager un pari: on peut, bien sûr, dès l'abord, en mesurer plus ou moins lucidement les termes, mais en définitive seul le temps permettra de dire s'il était ou non fondé.

Alors, il faut accepter l'incertitude; elle est le lot inévitable de toute entreprise naissante. Nous le faisons avec confiance. Parce que nous avons la conviction que la R.G.N. répond vraiment à une attente, à un besoin, et que notre pari a finalement toutes les chances d'être gagné.

En matière de publications nucléaires, la situation actuelle, dans notre pays, n'est guère satisfaisante. Telle est du moins l'opinion de nombreux chercheurs et techniciens qui estiment que la multiplicité des revues étrangères: allemandes, américaines, anglaises, soviétiques, ne peut compenser tout à fait l'absence d'une grande revue nucléaire de langue française. C'est pour combler ce vide que nous avons créé la R.G.N., en espérant qu'elle deviendra, pour tous ceux qui, en France, participent au développement de la science et "de la technologie nucléaires, le grand organe d'information, de liaison, de référence.

Si nous avons l'ambition de faire de la R.G.N. une publication de haut niveau scientifique destinée aux chercheurs, aux techniciens, aux industriels, nous voulons aussi l'ouvrir à un public plus large. C'est la dimension même du «fait nucléaire" - dont les implications intéressent maintenant l'ensemble de la communauté nationale - qui nous y invite. Aussi bien souhaitons-nous publier de nombreux articles et informations accessibles aux non-spécialistes. Nous entendons de même prendre notre part, dans un esprit de dialogue constructif et de respect de tous les points de vue, à tous les débats concernant l'utilisation pacifique de l'énergie nucléaire et intéressant l'avenir économique et industriel de notre pays.

Voilà donc, brièvement présentées, les intentions; il reste à les mettre en œuvre... Tous ceux qui s'intéressent, à des titres divers, aux problèmes nucléaires peuvent et doivent nous apporter leurs conseils, leurs suggestions, leur concours. De la part des membres de la Société Française d'Énergie Nucléaire, ils nous sont d'ores et déjà acquis. C'est en effet la S.F.E.N. - créée en novembre 1973 - qui a eu l'initiative de cette revue; c'est à elle que revient la responsabilité de l'animer. Pour tous ses adhérents, chercheurs, ingénieurs, techniciens, médecins, professeurs... dont l'objectif commun est l'avancement de la science et de la technique nucléaires, la R.G.N. constituera un lieu privilégié d'expression et d'information.

A tous les autres, nous disons que cette revue est aussi la leur. Notre philosophie n'est pas celle de la tour d'ivoire ou du splendide isolement, mais celle de la communication et de l'échange. C'est avec vous tous, auteurs et lecteurs, que nous voulons modeler le visage de la R.G.N. pour en faire le grand organe d'information à la mesure du «fait nucléaire» français.

Rémy CARLE

Président de la Société Française d’Énergie Nucléaire. 